\title{
Nuclear Factor Erythroid 2-Related Factor 2 (Nrf2) Regulates Epidermal Keratinization under Psoriatic Skin Inflammation
}

Tatsuya Ogawa, Yosuke Ishitsuka, Sae Inoue, Yoshiyuki Nakamura, Akimasa Saito, Naoko Okiyama, Yasuhiro Fujisawa, Junichi Furuta, Rei Watanabe, and Manabu Fujimoto

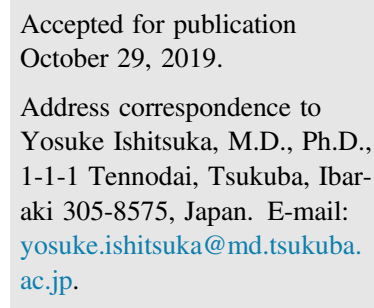

From the evolutionary and adaptive perspectives of the skin, timely neutrophil recruitment in response to tissue-derived danger signals is instrumental in eradicating microorganisms on the surface. ${ }^{1}$ However, an inflammatory response involving excessive neutrophil recruitment leads to uncontrolled production of reactive oxygen species and autoinflammatory/ autoimmune tissue destruction, a typical pathologic consequence of which is psoriasis. The transcription factor nuclear factor erythroid 2-related factor 2 (NRF2) and its cytoskeletonassociated negative regulator Kelch-like erythroid cell-derived protein with cap-n-collar homology-associated protein 1 (KEAP1) system constitutes a master regulator of an antioxidant/antielectrophilic response, ${ }^{2}$ and $\mathrm{Nrf2}$-deficient $\left(\mathrm{Nrf} 2^{-/-}\right)$ mice not only show dysregulated innate immune responses ${ }^{3,4}$ but also develop autoimmune phenotypes. ${ }^{5}$ Conversely, constitutive activation of NRF2, which can be obtained by inhibiting the negative regulator KEAP1, protects against various forms of inflammation, such as lipopolysaccharideinduced septic shock, ${ }^{4}$ acute kidney injury, ${ }^{6}$ and lethal autoimmune phenotypes. ${ }^{7}$ In fact, Keapl-deficient mice suffer from hyperorthokeratosis in the forestomach that leads to lethality, and Nrf2 directly transactivates the gene that encodes for loricrin (Lor), ${ }^{8}$ a keratohyalin granule constituent that mediates

Supported by a JSPS KAKENHI grant, Grant-in-Aid for Research Activity Start-up 16H06663 (Y.I.), and Early-Career Scientists grant 18K16018 (Y.I.).

Disclosures: None declared. 
cornification by cross-linking cytoskeletal proteins keratin 1/10 (K1/K10) and filaggrin (Flg). ${ }^{9}$ The recovery of epidermal barrier function in the absence of Lor requires KEAP1/NRF2 system activation. ${ }^{10,11}$ These lines of evidence underscore that NRF2 is a regulator of epidermal barrier function. ${ }^{8,12}$

Typical psoriasis plaques form well-demarcated parakeratotic lesions, and noninflamed (nonlesional) areas show orthokeratosis; these mutually exclusive modes of keratinization are hallmarks of psoriasiform inflammation and quasihomeostasis, respectively. Moreover, dimethyl fumarate (DMF), an electrophilic reagent, has been used for the treatment of psoriasis $^{13,14}$ and recently became a Food and Drug Administration-approved drug for the treatment of relapsing-remitting multiple sclerosis. ${ }^{15}$ Therefore, it is hypothesized that NRF2 not only regulates inflammatory responses but also is required for the maintenance of orthokeratosis in an inflammatory epidermal milieu. Here, $N r f 2^{-1-}$ mice show an enhanced inflammatory response and parakeratosis in imiquimod (IMQ)-induced cutaneous inflammation, which is a model of the psoriasiform tissue reaction. ${ }^{16}$

\section{Materials and Methods}

Mice

C57BL/6 mice (age, 8 to $12 \mathrm{wk}$ ) were obtained from Charles River Laboratories Japan (Yokohama, Japan), and $N r f 2^{-1-}$ mice were from Riken Bioresource Research Center (Tsukuba, Japan). ${ }^{17}$ All mice were maintained under specific pathogen-free conditions at the animal facility of the University of Tsukuba (Tsukuba, Ibaraki, Japan), and all procedures were approved by the University of Tsukuba Ethics Committee.

\section{IMQ-Induced Psoriasis-Like Skin Inflammation Model}

Eight-week-old $\mathrm{Nrf2^{-/- }}$ and wild-type $\left(\mathrm{Nrf2^{+/+ }}\right)$ female mice with the C57BL/6 background were used. On day 0 , the back skin was trimmed carefully using a mechanical trimmer (Trimmer 8655; Wahl, Sterling, IL), and $60 \mathrm{mg} /$ day (back, $50 \mathrm{mg}$; ear, $10 \mathrm{mg}$ ) of $5 \%$ IMQ cream (Mochida Pharmaceutical, Tokyo, Japan) was applied.

\section{N-Acetylcysteine Treatment}

$\mathrm{N}$-acetylcysteine (NAC) was purchased from FUJIFILM Wako Pure Chemical Corporation (Osaka, Japan). The mice received either deionized water or water containing NAC (7 $\mathrm{mg} / \mathrm{mL}$; neutralized to $\mathrm{pH} 7.4$ with $\mathrm{NaOH}$ ) starting 2 days before the IMQ treatment until termination. Water containing NAC was prepared freshly every day.

\section{DMF Treatment}

DMF was purchased from FUJIFILM Wako Pure Chemical Corporation. The reagent was diluted in $200 \mu \mathrm{L}$ of $0.1 \%$
Methocel (FUJIFILM Wako Pure Chemical Corporation)/ $\mathrm{H}_{2} \mathrm{O}$ and administered by oral gavage starting from day 0 until termination. The mice received DMF $(15 \mathrm{mg} / \mathrm{kg})$ or the vehicle control $\left(\right.$ Methocel $\left./ \mathrm{H}_{2} \mathrm{O}\right)$ twice a day.

\section{Keratinocyte Culture and Knockdown Experiment}

Neonatal human epidermal keratinocytes (C0015C; Thermo Fisher Scientific, Waltham, MA) were maintained in EpiLife medium (MEPI500CA; Thermo Fisher Scientific) with EpiLife Defined Growth Supplement (s0125; Thermo Fisher Scientific). Lentiviral particles encoding shRNA targeting Keapl mRNA were purchased from Santa Cruz Biotechnology (Dallas, TX; sc-43878-V). Lentiviral particles encoding a scrambled shRNA sequence (Scr) (sc-108080; Santa Cruz Biotechnology) also were purchased for use as a control. Neonatal human epidermal keratinocyte cells were transduced with these lentiviral particles in accordance with the manufacturer's instructions, and the knockdown efficacy was assessed at the mRNA level by quantitative real-time PCR. For the analysis of the proliferation rate, neonatal human epidermal keratinocyte cells of each strain were seeded into 6-well plates at a density of $5 \times 10^{4}$ cells/well in the EpiLife medium. The next day, the medium was changed for fresh medium, and cell numbers in triplicate wells were counted by trypsinization at the indicated time points.

\section{Generation of Bone Marrow Chimera Mice}

To generate bone marrow chimera mice, recipient $\mathrm{Nrf} 2^{+/+}$ or $N r f 2^{-1-}$ mice underwent 9-Gy total body irradiation on day 1. Bone marrow single-cell suspensions from either $\mathrm{Nrf2} 2^{+/+}$or $\mathrm{Nrf} 2^{-/-}$mice on day 0 intravenously through the tail vein, and mice that had undergone mock injection failed to survive 7 days after the irradiation, validating successful bone marrow suppression. Successful generation of chimerism was determined through the genotyping of DNA derived from peripheral blood and tail snip. The mice received sterilized water acidified with hydrochloric acid for 2 months after transplantation to prevent opportunistic infection.

\section{Collection of Lesional and Nonlesional Skin of Human Psoriasis}

Biopsy samples from lesions and normal-looking nonlesional skin were taken from patients with psoriasis using a 3 -mm punch. Pieces of skin samples were homogenized in TRIzol reagent (15596-026; Thermo Fisher), and total RNA was isolated. Written informed consent was provided from all subjects under the approval from the Institutional Review Board at the University of Tsukuba (Tsukuba Clinical Research and Development Organization; H29-003). 
Table 1 Predesigned Quantitative PCR Assay

\begin{tabular}{ll}
\hline Gene & Assay ID \\
\hline Il6 & Mm.PT.58.10005566 \\
Inf & Mm.PT.58.12575861 \\
Il23a & Mm.PT.58.10594618.g \\
Il17a & Mm.PT.58.6531092 \\
Flg & Mm.PT.58.29195892 \\
Lor & Mm.PT.58.12882060 \\
KEAP1 & Hs.PT.58.1898764 \\
NRF2 & Hs.PT.58.28159373 \\
NQ01 & Hs.PT.58.2697277 \\
GSTP1 & Hs.PT.58.406347 \\
HMOX1 & Hs.PT.58.45340055 \\
LOR & Hs.PT.58.27761511 \\
SPRR2A & Hs.PT.58.19341995 \\
FLG & Hs.PT.58.24292320.g \\
\hline
\end{tabular}

The predesigned quantitative PCR assay was from Integrated DNA Technologies (Coralville, IA).

\section{Histopathology and Immunohistochemistry}

The ear or back skin was fixed overnight with $10 \%$ buffered formalin. After paraffin embedding, $3-\mu \mathrm{m}$ sections were deparaffinized and incubated overnight with primary antibodies diluted as follows after routine retrieval and blocking: anti-proliferating cell nuclear antigen $(0.14 \mu \mathrm{g} / \mathrm{mL}$, GTX100539; GeneTex, Irvine, CA), anti-STAT3 (0.6 $\mu \mathrm{g} /$ mL, GTX104616; GeneTex), anti-phosphorylated STAT3 $(0.6 \mu \mathrm{g} / \mathrm{mL}$, GTX118000; GeneTex), anti-FLG $(0.2 \mu \mathrm{g} / \mathrm{mL}$, PRB-417P-050; Eurogentec, Seraing, Belgium), anti-LOR (1:1000; the antibody was kindly provided by Dr. Dennis R. Roop, University of Colorado) ${ }^{18}$ anti-K5 $(0.1 \mu \mathrm{g} / \mathrm{mL}$, PRB160P; BioLegend), anti-K6 (1:1000, the antibody was kindly provided by Dr. Dennis R. Roop), ${ }^{19}$ anti-K10 (0.048 $\mu \mathrm{g} / \mathrm{mL}$, ab76318; Abcam, Cambridge, England), anti-K16 (1 $\mu \mathrm{g} / \mathrm{mL}$, CSB-PA002020; Wuhan Huamei, Wuhan, China), and anti-K17 (1 $\mu \mathrm{g} / \mathrm{mL}$, CSB-PA002024; Wuhan Huamei). After blocking of endogenous peroxidase with PBS containing $0.3 \% \mathrm{NaN}_{3}$ and $0.01 \% \mathrm{H}_{2} \mathrm{O}_{2}$, visualization was performed using an $\mathrm{ABC}$ system according to the standard protocol (Vector Laboratories, Burlingame, CA), followed by light counterstaining with hematoxylin. Proliferating cell nuclear antigen, STAT3, and phosphorylated STAT3-positive keratinocytes were counted in highpower fields by three independent dermatologists (T.O., Y.N., and Y.I.) using a digital microscope (AX80; Olympus, Tokyo, Japan) and Flovel Filing System (Flovel, Tokyo, Japan), and the positivity rate for each immunogen was subjected to statistical analysis. The expression levels of LOR FLG, K5, K6, K16, and K17 were evaluated according to the staining intensity of the positive keratinocytes as follows: grade 1, none; grade 2, weak; grade 3, strong; and grade 4, very strong. The expression level of K10 was evaluated according to the numbers of K10-negative layers as follows: grade 1, all layers; grade 2, 5 to 10 layers; grade
3, 2 to 4 layers; and grade 4, one layer, as described previously. $^{20}$

\section{Quantitative Real-Time PCR}

Pieces of full-thickness skin were homogenized in TRIzol reagent (15596018; Thermo Fisher), and total RNA was isolated. cDNA was synthesized using the High-Capacity cDNA Reverse Transcription Kit (4368814; Thermo Fisher). Primers specific for each target with double-quenched probes were purchased from the IDT Primetime Predesigned Library (IDT, Coralville, IA) (Table 1), and primers for Gapdh (4352339E; Thermo Fisher) were used for internal reference. All data were analyzed using the Applied Biosystems 7500 Fast Real-Time PCR System (Thermo Fisher). The interpretation of quantitative real-time PCR data was conducted using the comparative $\mathrm{Ct}$ method ( $\Delta \Delta \mathrm{CT}$ method).
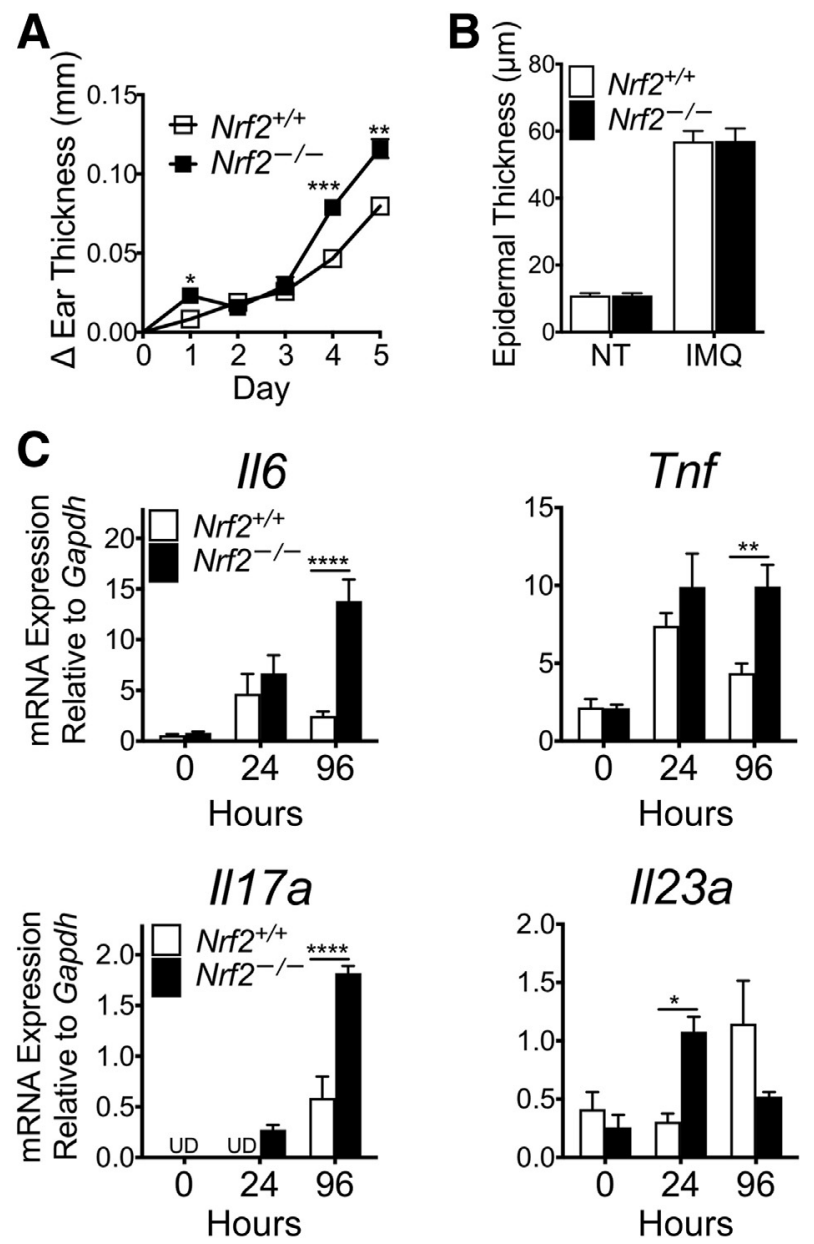

Figure 1 A: Ear thickness of $\mathrm{Nrf2}^{-/-}$and $\mathrm{Nrf2}^{+/+}$mice treated with $5 \%$ imiquimod (IMQ) cream for 5 consecutive days.B: Epidermal thickness of nontreated (NT) or IMQ-treated back skin on day 5. The thickness was measured by calculating the distance between the basal layer and the top of the granular layer. C: mRNA expression levels of psoriasis-related cytokine genes at the indicated time points in the ear skin. $n=6$. ${ }^{*} P<0.05$, ${ }^{* *} P<0.01,{ }^{* * *} P<0.005$, and ${ }^{* * *} P<0.001$ versus $N r f 2^{+/+}$. UD, undetected. 


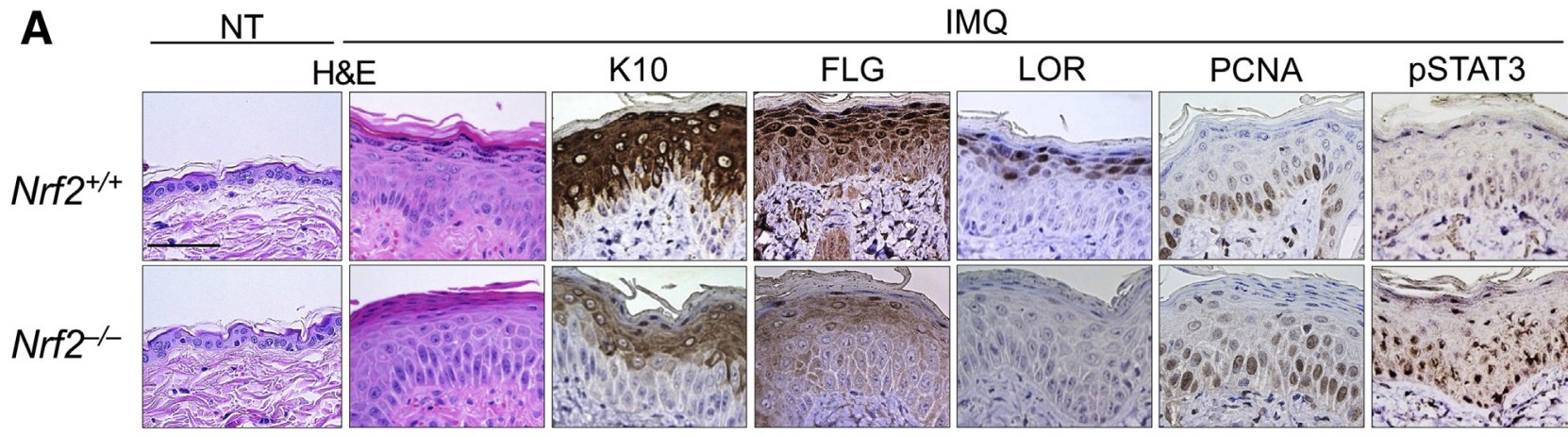

B

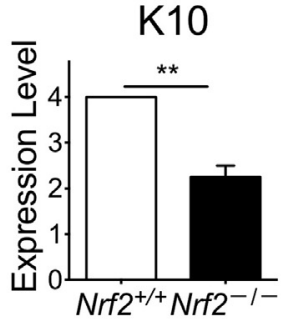

K16

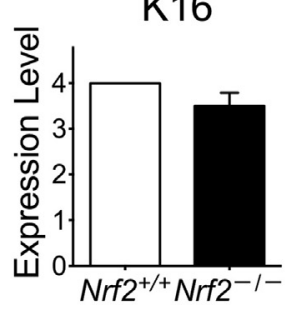

FLG

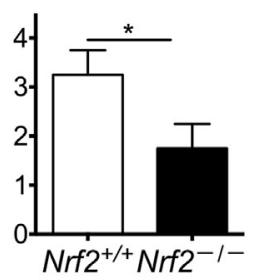

K17

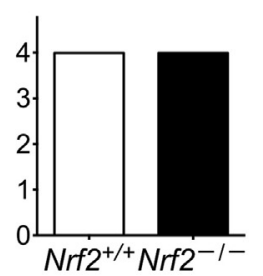

LOR

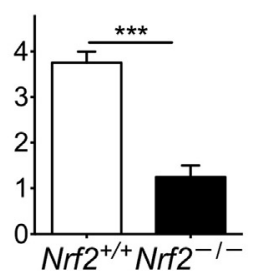

PCNA

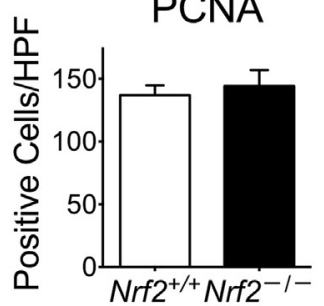

K5

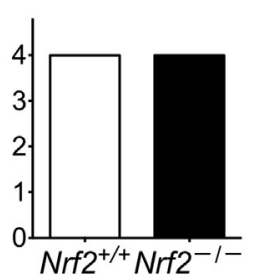

STAT3

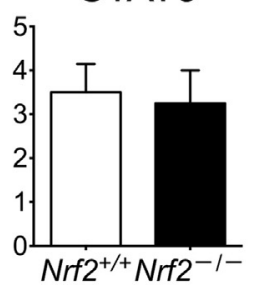

K6
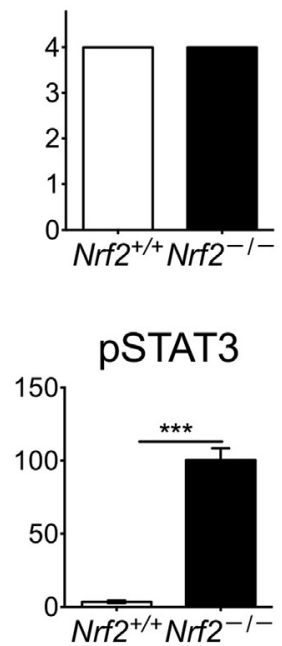

Figure 2 A: Histologic images of nontreated (NT) or imiquimod (IMQ)-treated back skin for 5 consecutive days. Representative images of hematoxylin and eosin (H\&E) staining or immunohistochemistry for keratin 10 (K10), filaggrin (FLG), loricrin (LOR), proliferating cell nuclear antigen (PCNA), and phosphorylated STAT3 (pSTAT3) on IMQ-treated back skin. B: Protein expression levels as determined by the number of negative layers (K10), staining intensity (LOR, FLG, K5, and K6; staining intensity in the epidermis), or positivity rate [PCNA, STAT3, and PSTAT3; positive keratinocytes per high-power field (HPF)]. $n=4 .{ }^{*} P<0.05,{ }^{* *} P<0.01$, and ${ }^{* *} P<0.005$. Scale bars $=50 \mu \mathrm{m}$.

\section{Statistical Analysis}

Data are expressed as means \pm SEM. Comparisons were performed using the unpaired $t$-test with the Welch correction (between two groups) or the two-way analysis of variance (time-course experiments) using Prism 7 software (GraphPad, La Jolla, CA). In all analyses, $P<0.05$ was taken to indicate statistical significance.

\section{Results}

\section{Nrf2 Attenuates IMQ-Induced Skin Inflammation}

$N r 2^{-/-}$mice were subjected to daily application of IMQ cream and showed significantly enhanced ear swelling compared with wild-type $\left(\mathrm{Nrf}^{+/+}\right)$mice (Figure 1A). Accordingly, the expression levels of the psoriasis-related proinflammatory cytokine genes were increased significantly in $\mathrm{Nrf2^{-/- }}$ mice compared with those in $\mathrm{Nrf2^{+/+ }}$ mice after IMQ treatment in the ear skin. Reflecting the activation of leukocytes with dendritic cell/monocytes lineage in response to danger signals, ${ }^{1} I l 23$ expression levels were significant at the earlier time point, whereas significantly increased expression levels of $I l 6, T n f$, and $I l 17$ were noted at the later time point (Figure 1C). However, the expression levels of $I l 22$ were not changed substantially in $N r f 2^{-1-}$ mice compared with those in $\mathrm{Nrf}^{+/+}$mice (data not shown), and epidermal hyperplasia (acanthosis) was evoked to comparable degrees in both genotypes (Figures 1B and 2). Correspondingly, proliferating cell nuclear antigen staining signals, which denote DNA replication activity, were not altered substantially between the genotypes (Figure 2).

\section{Nrf2 Attenuates Psoriasiform Tissue Reaction}

The epidermal phenotypes in IMQ-treated back skin were analyzed. IMQ-treated $\mathrm{Nrf}^{-/-}$back skin epidermis not only had obvious edema (spongiosis) but also showed prominent hypogranulosis and parakeratosis, the hallmarks 
A

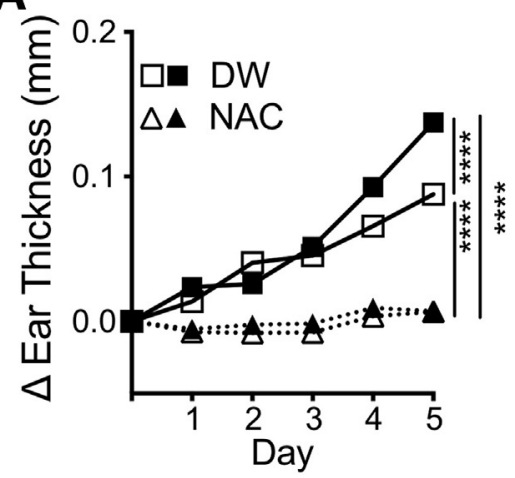

B

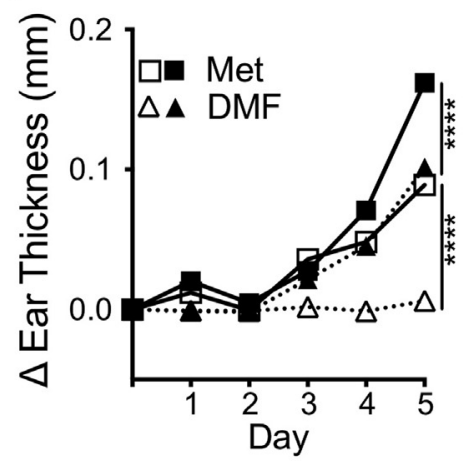

C
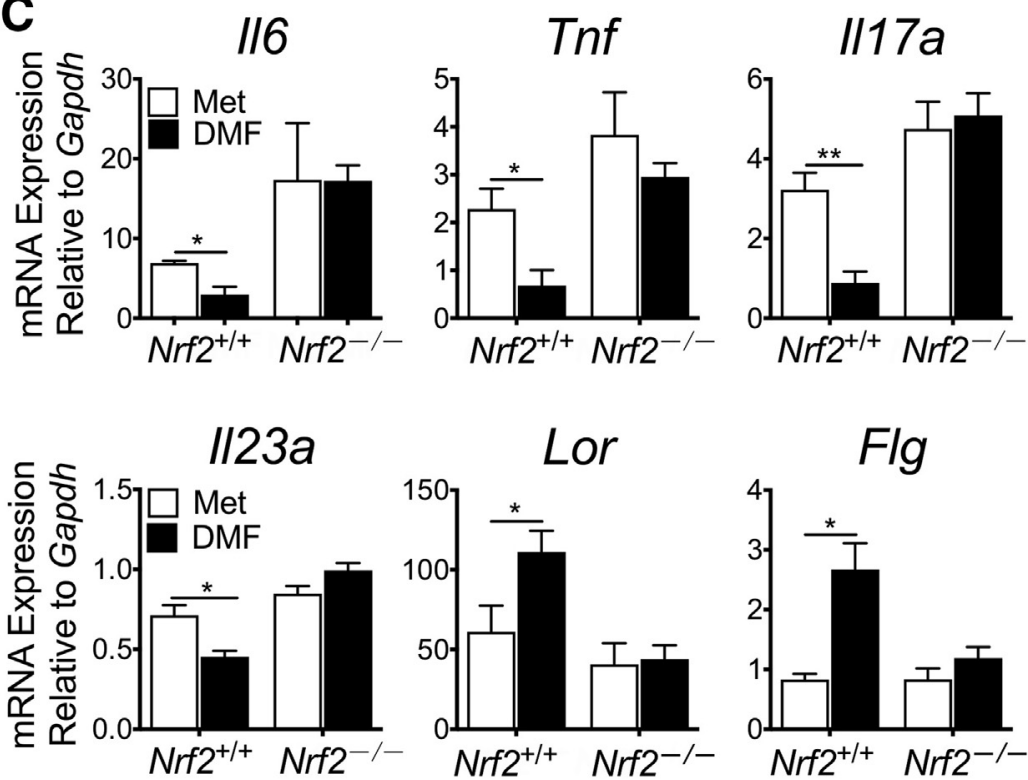

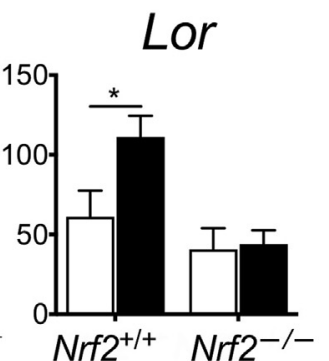

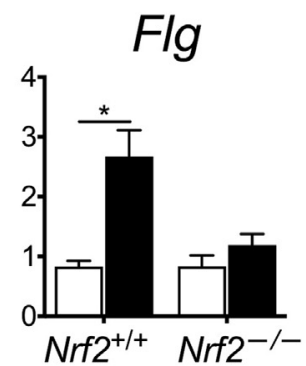

Figure 3 A: Ear thickness of $\mathrm{Nrf2}^{-/-}$and $\mathrm{Nrf2}^{+/+}$mice treated with $5 \%$ imiquimod (IMQ) cream for 5 consecutive days with intervention of either deionized water (DW) or $\mathrm{N}$-acetylcysteine (NAC). Closed triangles indicate NACtreated $\mathrm{Nrf2}^{-/}$mice; open triangles, NAC-treated $\mathrm{Nrf2}^{+/+}$mice; closed squares, control-treated $\mathrm{Nrf2}^{-1-}$ mice; open squares, control-treated $\mathrm{Nrf2}^{+/+}$mice. B: Ear thickness of $\mathrm{Nrf2}^{-/-}$and $\mathrm{Nrf2}^{+/+}$mice treated with $5 \%$ IMQ cream for 5 consecutive days with intervention of either vehicle [Methocel (Met)] or dimethyl fumarate (DMF). Closed triangle, DMF-treated $\mathrm{Nrf2}^{-/-}$mice; open triangle, DMF-treated $\mathrm{Nrf2}^{+/+}$mice; closed square, vehicle-treated $\mathrm{Nrf2}^{-/-}$mice; open square, vehicletreated $\mathrm{Nrf2}^{+/+}$mice. C: mRNA expression levels of psoriasis-related cytokine and epidermal differentiation complex genes on day 4. $n=6$ (A and B); $n=4$ (C). ${ }^{*} P<0.05,{ }^{* *} P<0.01,{ }^{* * * *} P<0.001$. of psoriasiform tissue reaction. ${ }^{15}$ On the other hand, IMQtreated $\mathrm{Nrf} 2^{+/+}$mice retained stratum granulosum and orthokeratosis despite the presence of acanthosis (Figure 2). Reflecting the absence of stratum granulosum, immunohistochemical analysis showed significantly decreased expression levels of LOR, FLG, and the early keratinization marker K10, whereas the expression levels of basal keratin K5 and stress-induced keratins K6/K16/K17 were unchanged between the two genotypes (Figure 2). Staining signals of the phosphorylated STAT3 protein, ${ }^{21}$ but not unphosphorylated STAT3 protein, in IMQ-treated $\mathrm{Nrf}^{-1-}$ epidermis were comparable with those in the $\mathrm{Nrf2}^{+/+}$ counterpart (Figure 2).

Reversal of IMQ-Induced Skin Inflammation with the Thiol Antioxidant NAC

KEAP1-inhibition-mediated NRF2 activation profoundly depends on the modification of sulfhydryl groups on cysteine residues of the KEAP1 protein that are oxidized by reactive oxygen species, ${ }^{22}$ and free sulfhydryl groups were supplemented by way of oral NAC treatment. As expected, NAC treatment completely abrogated the ear swelling response in both genotypes (Figure $3 \mathrm{~A}$ ).

\section{DMF Attenuates Psoriasiform Tissue Reaction}

To determine the therapeutic efficacy of DMF in the experiment, the mice were treated with DMF by oral gavage, as previously described. ${ }^{23}$ DMF treatment in $\mathrm{Nrf2} 2^{+\prime}$

${ }^{+}$did not evoke an ear swelling response, while $N r f 2^{-/-}$ mice ended up with a partial attenuation (Figure 3B). In $N r f 2^{+/+}$mice, the expression levels of Il6, Tnf, Ill7a, and Il23a were decreased significantly in response to DMF treatment (Figure 3C), but those of Il22 were not decreased in the DMF-treated group compared with the vehicle control group (data not shown). Accordingly, although the degree of acanthosis was not affected in response to the treatment, the expression levels of Lor and Flg were increased significantly compared with those in the vehicle-treated $N r 2^{+/+}$mice, and changes in the expression levels of the inflammatory cytokines and the epidermal differentiation 

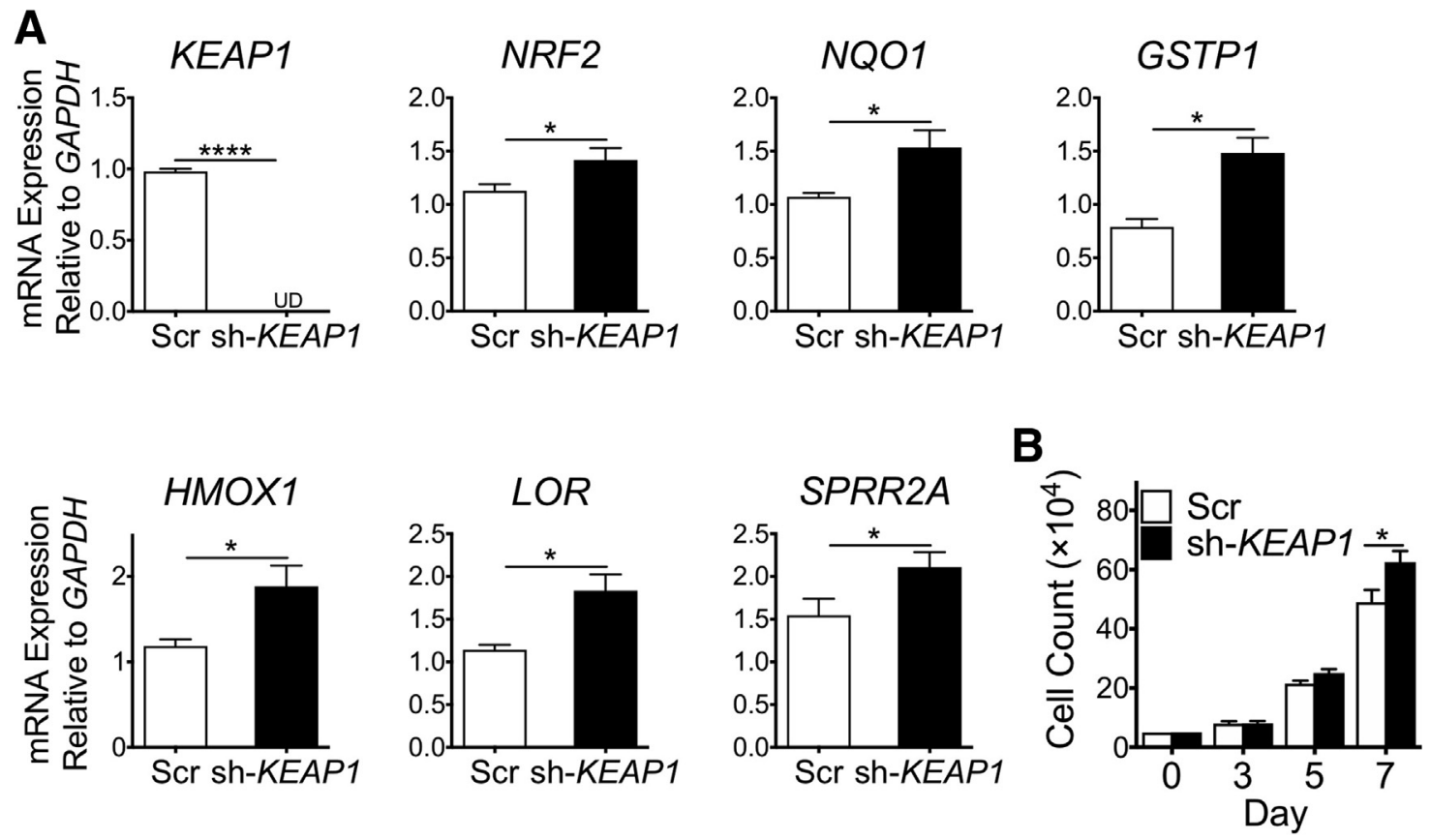

C

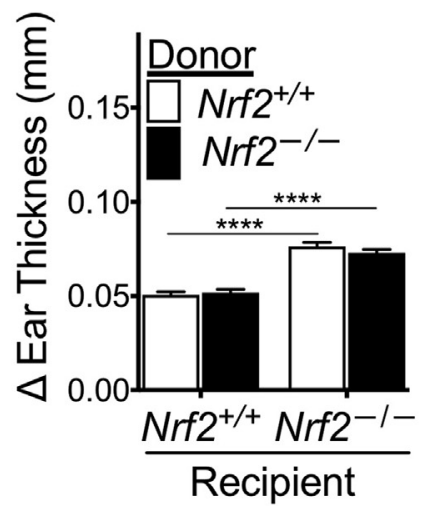

$\mathbf{D}$

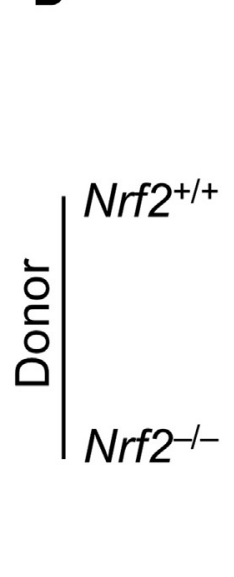

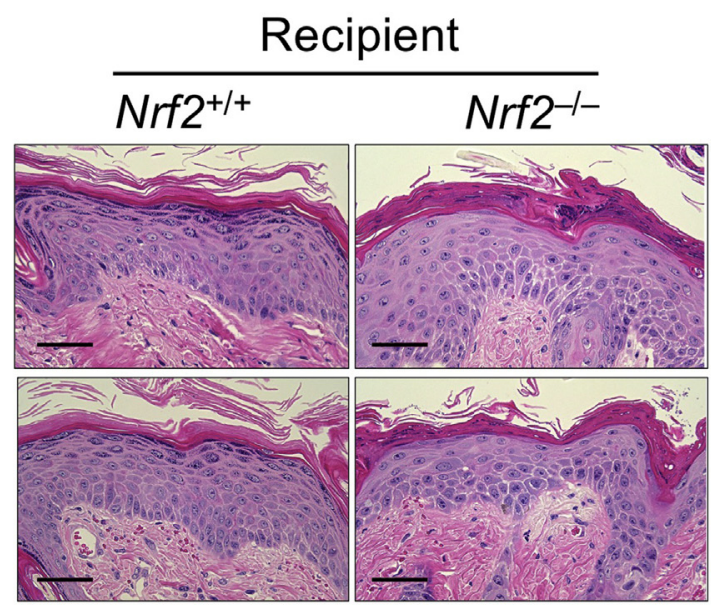

Figure 4 A: Neonatal human epidermal keratinocyte cells were cultured under proliferative conditions $\left(0.06 \mathrm{mmol} / \mathrm{L} \mathrm{Ca}^{2+}\right)$, and $K E A P 1 \mathrm{knockdown}$ experiments were performed to activate the KEAP1/NRF2 system. Efficient knockdown was validated at the mRNA level. KEAP1 knockdown enhances NRF2 and downstream target genes expression levels. Results are from at least three independent experiments. B: A proliferation assay was performed to analyze the effect of KEAP1 knockdown-mediated NRF2 overexpression. Results are from at least three independent experiments. C and $\mathbf{D}$ : Nrf2 ${ }^{-/-}$or Nrf2 $2^{+/+}$mice underwent 9-Gy total body irradiation and received bone marrow single-cell suspensions from $\mathrm{Nrf2}^{-/-}$or $\mathrm{Nrf2}^{+/+}$mice. Two months after transplantation, each chimera mouse was treated with $5 \%$ imiquimod cream for 5 consecutive days. C: Ear thickness of each group on day 5 . D: Representative images of hematoxylin and eosin staining. $n=4(\mathbf{A}) ; n=6$ (B and C). Scale bars $=50 \mu \mathrm{m} .{ }^{\star} P<0.05,{ }^{*} * \star * P<0.001$. Scr, scramble; UD, undetected.

complex genes in response to the treatment were attenuated in $\mathrm{Nrf2}^{-/-}$mice (Figure 3C).

Nrf2 Activates the Antioxidative Response in the Epidermis

$N R F 2$ is known to enhance keratinocyte proliferation, and its expression is enhanced in psoriatic epidermis compared with normal skin. ${ }^{24} \mathrm{~A}$ knockdown approach was taken to activate the KEAP1/NRF2 system. Knockdown of KEAP1 using a shRNA-encoding lentiviral vector resulted in significantly increased mRNA expression levels of NRF2 and its downstream target genes ${ }^{2} \mathrm{NAD}(\mathrm{P}) \mathrm{H}$ (nicotinamide-adenine dinucleotide phosphate, reduced form) dehydrogenase, quinone 1 (NQO1), glutathione S-transferase pi 1 (GSTP1), heme oxygenase 1 (Hmox1), LOR, and small proline-rich protein 2A (SPRR2A) (Figure 4A). Significantly increased proliferation rates also were noted (Figure 4B). Psoriasiform tissue reaction requires blood flow-derived neutrophils as primary effector cells. ${ }^{1,16}$ Given the pleiotropic role of Nrf2 to counteract the inflammatory response and NRF2 activation in psoriatic epidermis, the results described earlier in this paragraph suggest that keratinocyte hyperproliferation in psoriatic epidermis was a consequence of the host's inherent 

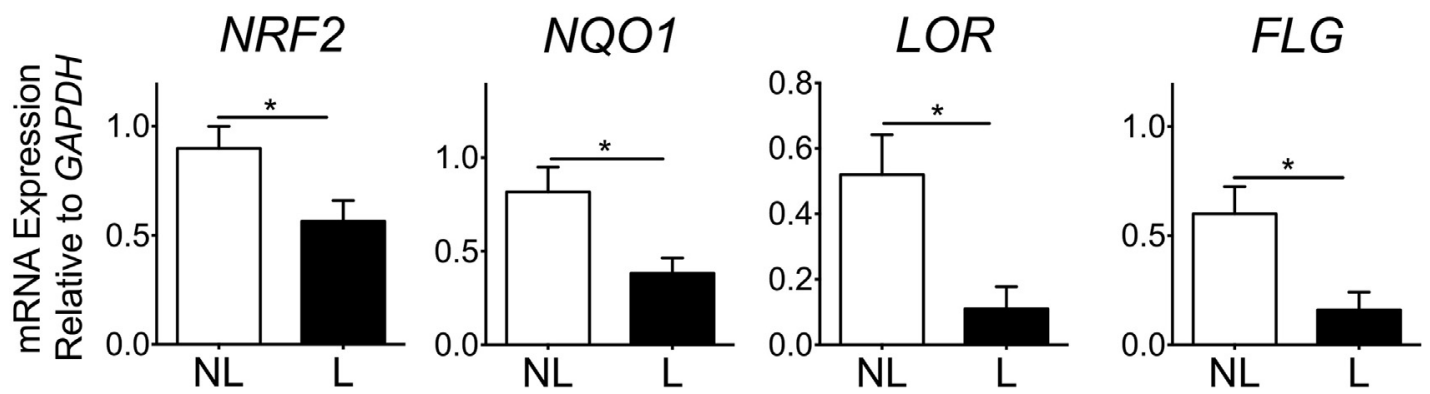

Figure 5 Expression levels of NRF2, NQ01, LOR, and $F L G$ in lesional (L) or nonlesional (NL) skin of psoriasis patients at the mRNA level. $n=8 .{ }^{*} P<0.05$.

anti-inflammatory response on the KEAP1/NRF2 system. To address the issue, bone marrow chimeras were generated in which bone marrow cells from each genotype were transplanted into either genotype. $\mathrm{Nrf2^{-/- }}$ recipients not only showed significantly increased ear swelling (Figure 4C), but also showed remarkable hypogranulosis and parakeratosis compared with $\mathrm{Nrf}^{+/+}$recipients, whereas the $\mathrm{Nrf2^{-/- }}$ donor did not (Figure 4D). This indicated that radioresistant cells such as keratinocytes or IL-17-producing dermal $\gamma \delta \mathrm{T}$ cells $^{25,26}$ are responsible for the enhanced psoriasiform tissue reaction observed in $\mathrm{Nrf}^{-/-}$mice. Collectively, these results indicated that Nrf2 activates an antioxidative response in the epidermis.

\section{Decreased NRF2 and Downstream Target Genes in Lesional Psoriasis Skin}

Considering that the activation of the KEAP1/NRF2 system attenuated psoriasiform tissue reaction, the expression levels of NRF2 target genes were analyzed in skin samples from patients with psoriasis. As expected, the mRNA expression levels of $N R F 2$ and its downstream targets $N Q O 1,{ }^{2}$ and $L O R,{ }^{8}$ as well as $F L G$, were decreased significantly in the lesional area compared with the nonlesional area (Figure 5), validating the dysregulated KEAP1/NRF2 system that leads to the persistent psoriasiform tissue reaction that is critical for psoriatic plaque generation.

\section{Discussion}

Immune therapy targeting IL-17 or its leukocyte-derived upstream cytokine IL-23 has brought a remarkable treatment efficacy and safety for psoriasis and related conditions. ${ }^{27}$ However, despite mechanistic evidence from a mouse model, ${ }^{28}$ targeting IL-22, another IL-23 downstream cytokine that mediates epidermal hyperproliferation after IL-23-induced STAT3 activation, ${ }^{29}$ was not effective. ${ }^{30}$ This unequivocally suggests that the IL-23/IL-17 pathway primarily mediates the psoriasiform tissue reaction, ${ }^{16}$ rather than mere acanthosis. Accordingly, DMF reversed the

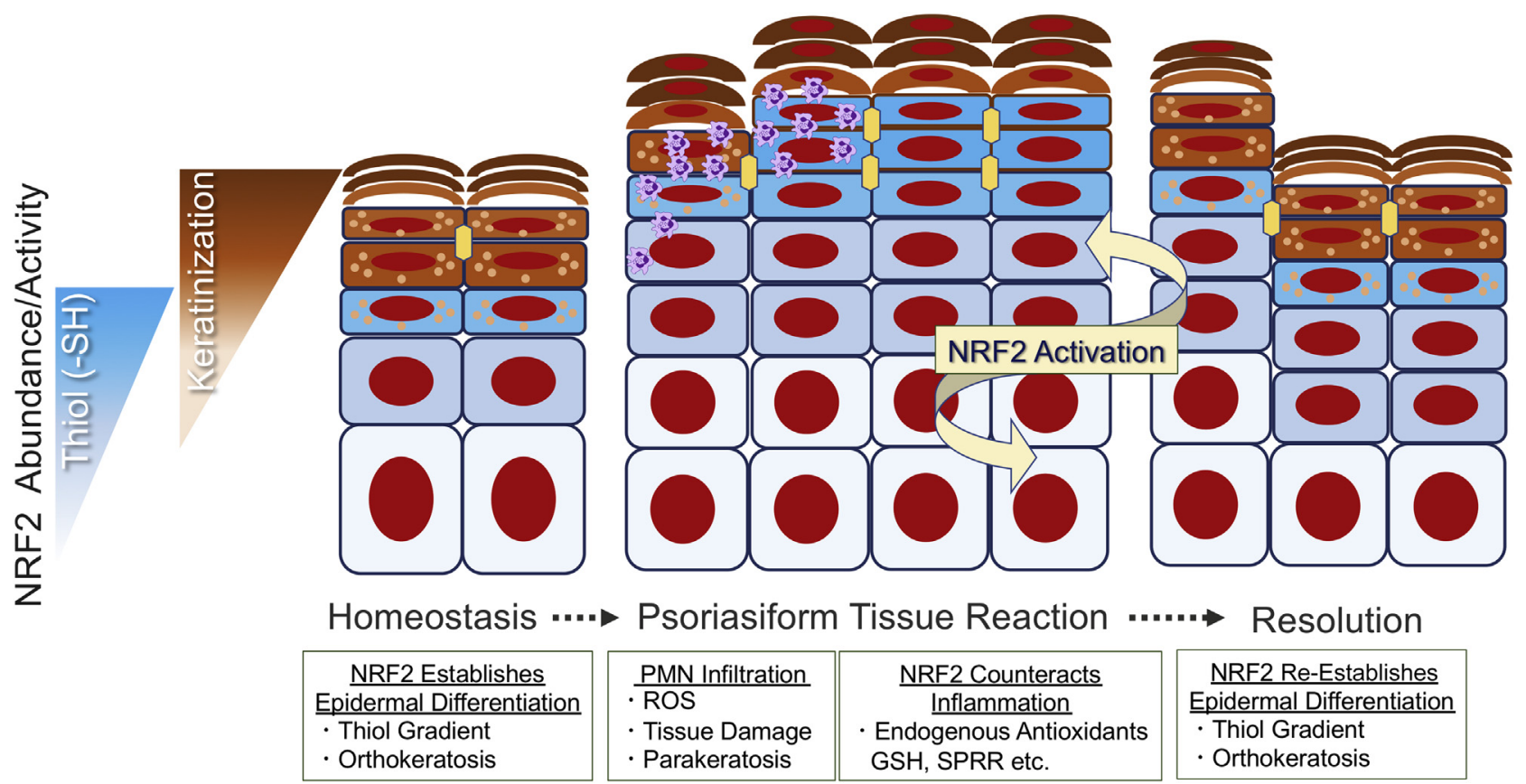

Figure 6 A schematic representation of the role of the KEAP1/NRF2 system that regulates epidermal homeostasis during the psoriasiform tissue reaction. GSH, glutathione; PMN, polymorphonuclear neutrophil; ROS, reactive oxygen species; -SH, thiol; SPRR, small proline-rich. 
expression levels of the effector cytokine $I l 17$, but not $I l 22$, in vivo in an Nrf2-dependent manner. NRF2 directly transactivates the stress-related cytokeratins $\mathrm{K} 6 / \mathrm{K} 17^{8,24,31}$ in response to abundant IL-17 and mediates keratinocyte hyperproliferation in lesional psoriasis skin. ${ }^{24}$ Therefore, hyperkeratosis after acanthosis presumably reflects an innate cytoprotective response against the excessive psoriasiform tissue reaction in the epidermis, which depends on the KEAP/NRF2 system (Figure 6).

Mrowietz and Asadullah ${ }^{13}$ proposed earlier that fumaric acid esters including DMF interfere with cellular redox systems and inhibit NF- $\kappa \mathrm{B}$ translocation, NRF2 counteracts $N F-\kappa B$ activation and regulates the innate immune response. $^{3}$ Indeed, autoinflammatory conditions in the epidermis, such as deficiency of the IL-1-receptor antagonist/IL-36-receptor antagonist ${ }^{32}$ or dominant gain-offunction caspase recruitment domain-containing protein $14,{ }^{33}$ result in psoriasis or generalized pustular psoriasis. Moreover, dendritic cells treated with the electrophile diethyl maleate ${ }^{34}$ or $\mathrm{DMF}^{35,36}$ retain immature/antiinflammatory phenotypes, and $N r f 2^{-/-}$dendritic cells show mature/proinflammatory phenotypes, ${ }^{37}$ which in turn profoundly affect the outcome of ensuing adaptive immune responses. Therefore, these results mechanistically corroborate the legitimacy of using DMF, an electrophilic drug, for the treatment of multiple autoinflammatory/autoimmune disorders in the epidermis, including psoriasis.

With this translational approach, NRF2 attenuates the psoriasiform tissue reaction, a primary inflammatory response in the surface barrier tissue, ${ }^{1}$ and therefore, the mechanistic legitimacy of using electrophilic drugs for the treatment of psoriasis and related conditions in which autoinflammation/autoimmunity plays significant roles is substantiated. However, given the immunosuppressive side effects in association with oral fumaric acid ester treatment that have been reported increasingly, ${ }^{38}$ the electrophilebased approach still may require a cautious optimization strategy.

\section{Acknowledgments}

We thank all of the patients with psoriasis who contributed to this study; Miwako Shobo, Yuriko Hirota, and Hideko Sakurai for helping with the laboratory work; and Dr. Dennis R. Roop (University of Colorado) for kindly providing the anti-LOR and anti-K6 antibodies.

\section{References}

1. McKenzie BS, Kastelein RA, Cua DJ: Understanding the IL-23-IL-17 immune pathway. Trends Immunol 2006, 27:17-23

2. Motohashi H, Yamamoto M: Nrf2-Keap1 defines a physiologically important stress response mechanism. Trends Mol Med 2004, 10: $549-557$

3. Thimmulappa RK, Lee H, Rangasamy T, Reddy SP, Yamamoto M, Kensler TW, Biswal S: Nrf2 is a critical regulator of the innate immune response and survival during experimental sepsis. J Clin Invest 2006, 116:984-995

4. Kong X, Thimmulappa R, Craciun F, Harvey C, Singh A, Kombairaju P, Reddy SP, Remick D, Biswal S: Enhancing Nrf2 pathway by disruption of Keap1 in myeloid leukocytes protects against sepsis. Am J Respir Crit Care Med 2011, 184:928-938

5. Yoh K, Itoh K, Enomoto A, Hirayama A, Yamaguchi N, Kobayashi M, Morito N, Koyama A, Yamamoto M, Takahashi S: Nrf2-deficient female mice develop lupus-like autoimmune nephritis. Kidney Int 2001, 60:1343-1353

6. Noel S, Martina MN, Bandapalle S, Racusen LC, Potteti HR, Hamad AR, Reddy SP, Rabb H: T lymphocyte-specific activation of Nrf2 protects from AKI. J Am Soc Nephrol 2015, 26:2989-3000

7. Suzuki T, Murakami S, Biswal SS, Sakaguchi S, Harigae H, Yamamoto M, Motohashi H: Systemic activation of NRF2 alleviates lethal autoimmune inflammation in scurfy mice. Mol Cell Biol 2017, 37

8. Wakabayashi N, Itoh K, Wakabayashi J, Motohashi H, Noda S, Takahashi S, Imakado S, Kotsuji T, Otsuka F, Roop DR, Harada T, Engel JD, Yamamoto M: Keap1-null mutation leads to postnatal lethality due to constitutive Nrf2 activation. Nat Genet 2003, 35: $238-245$

9. Rice RH, Durbin-Johnson BP, Ishitsuka Y, Salemi M, Phinney BS, Rocke DM, Roop DR: Proteomic analysis of loricrin knockout mouse epidermis. J Proteome Res 2016, 15:2560-2566

10. Huebner AJ, Dai D, Morasso M, Schmidt EE, Schafer M, Werner S, Roop DR: Amniotic fluid activates the nrf2/keap1 pathway to repair an epidermal barrier defect in utero. Dev Cell 2012, 23:1238-1246

11. Ishitsuka Y, Huebner AJ, Rice RH, Koch PJ, Speransky VV, Steven AC, Roop DR: Lce1 family members are Nrf2-target genes that are induced to compensate for the loss of loricrin. J Invest Dermatol 2016, 136:1656-1663

12. Schafer M, Farwanah H, Willrodt AH, Huebner AJ, Sandhoff K, Roop D, Hohl D, Bloch W, Werner S: Nrf2 links epidermal barrier function with antioxidant defense. EMBO Mol Med 2012, 4: 364-379

13. Mrowietz U, Asadullah K: Dimethylfumarate for psoriasis: more than a dietary curiosity. Trends Mol Med 2005, 11:43-48

14. Mrowietz U, Szepietowski JC, Loewe R, van de Kerkhof $P$, Lamarca R, Ocker WG, Tebbs VM, Pau-Charles I: Efficacy and safety of LAS41008 (dimethyl fumarate) in adults with moderate-tosevere chronic plaque psoriasis: a randomized, double-blind, Fumaderm ${ }^{\circledR}$ - and placebo-controlled trial (BRIDGE). Br J Dermatol 2017, 176:615-623

15. Tintore M, Sastre-Garriga J: Multiple sclerosis: dimethyl fumarate is coming of age. Nat Rev Neurol 2016, 12:436-437

16. Pinkus $\mathrm{H}$, Mehregan AH: The primary histologic lesion of seborrheic dermatitis and psoriasis. J Invest Dermatol 1966, 46:109-116

17. Itoh K, Chiba T, Takahashi S, Ishii T, Igarashi K, Katoh Y, Oyake T, Hayashi N, Satoh K, Hatayama I, Yamamoto M, Nabeshima Y: An Nrf2/small Maf heterodimer mediates the induction of phase II detoxifying enzyme genes through antioxidant response elements. Biochem Biophys Res Commun 1997, 236:313-322

18. Mehrel T, Hohl D, Rothnagel JA, Longley MA, Bundman D, Cheng C, Lichti U, Bisher ME, Steven AC, Steinert PM, Yuspa SH, Roop DR: Identification of a major keratinocyte cell envelope protein, loricrin. Cell 1990, 61:1103-1112

19. Imakado S, Bickenbach JR, Bundman DS, Rothnagel JA, Attar PS, Wang XJ, Walczak VR, Wisniewski S, Pote J, Gordon JS, Heyman RA, Evans RM, Roop DR: Targeting expression of a dominant-negative retinoic acid receptor mutant in the epidermis of transgenic mice results in loss of barrier function. Genes Dev 1995, 9: 317-329

20. Ishitsuka Y, Kawachi Y, Maruyama H, Taguchi S, Fujisawa Y, Furuta J, Nakamura Y, Ishii Y, Otsuka F: Pituitary tumor transforming gene 1 induces tumor necrosis factor-alpha production from keratinocytes: implication for involvement in the pathophysiology of psoriasis. J Invest Dermatol 2013, 133:2566-2575 
21. Sano S, Chan KS, Carbajal S, Clifford J, Peavey M, Kiguchi K, Itami S, Nickoloff BJ, DiGiovanni J: Stat3 links activated keratinocytes and immunocytes required for development of psoriasis in a novel transgenic mouse model. Nat Med 2005, 11:43-49

22. Itoh K, Wakabayashi N, Katoh Y, Ishii T, Igarashi K, Engel JD, Yamamoto M: Keap1 represses nuclear activation of antioxidant responsive elements by Nrf2 through binding to the amino-terminal Neh2 domain. Genes Dev 1999, 13:76-86

23. Linker RA, Lee DH, Ryan S, van Dam AM, Conrad R, Bista P, Zeng W, Hronowsky X, Buko A, Chollate S, Ellrichmann G, Bruck W, Dawson K, Goelz S, Wiese S, Scannevin RH, Lukashev M, Gold R: Fumaric acid esters exert neuroprotective effects in neuroinflammation via activation of the Nrf2 antioxidant pathway. Brain 2011, 134:678-692

24. Yang L, Fan X, Cui T, Dang E, Wang G: Nrf2 promotes keratinocyte proliferation in psoriasis through up-regulation of keratin 6, keratin 16, and keratin 17. J Invest Dermatol 2017, 137:2168-2176

25. Cai Y, Shen X, Ding C, Qi C, Li K, Li X, Jala VR, Zhang HG, Wang T, Zheng J, Yan J: Pivotal role of dermal IL-17-producing gammadelta T cells in skin inflammation. Immunity 2011, 35:596-610

26. Haas JD, Ravens S, Duber S, Sandrock I, Oberdorfer L, Kashani E, Chennupati V, Fohse L, Naumann R, Weiss S, Krueger A, Forster R, Prinz I: Development of interleukin-17-producing gammadelta T cells is restricted to a functional embryonic wave. Immunity 2012, 37:48-59

27. Hawkes JE, Chan TC, Krueger JG: Psoriasis pathogenesis and the development of novel targeted immune therapies. J Allergy Clin Immunol 2017, 140:645-653

28. Van Belle AB, de Heusch M, Lemaire MM, Hendrickx E, Warnier G, Dunussi-Joannopoulos K, Fouser LA, Renauld JC, Dumoutier L: IL22 is required for imiquimod-induced psoriasiform skin inflammation in mice. J Immunol 2012, 188:462-469

29. Zheng Y, Danilenko DM, Valdez P, Kasman I, Eastham-Anderson J, Wu J, Ouyang W: Interleukin-22, a T(H)17 cytokine, mediates IL-23induced dermal inflammation and acanthosis. Nature 2007, 445: 648-651

30. Conrad C, Gilliet M: Psoriasis: from pathogenesis to targeted therapies. Clin Rev Allergy Immunol 2018, 54:102-113
31. Kerns ML, Guss L, Fahey J, Cohen B, Hakim JM, Sung S, Lu RG, Coulombe PA: Randomized, split-body, single-blinded clinical trial of topical broccoli sprout extract: assessing the feasibility of its use in keratin-based disorders. J Am Acad Dermatol 2017, 76:449-453 e441

32. Cowen EW, Goldbach-Mansky R: DIRA, DITRA, and new insights into pathways of skin inflammation: what's in a name? Arch Dermatol 2012, 148:381-384

33. Jordan CT, Cao L, Roberson ED, Pierson KC, Yang CF, Joyce CE, Ryan C, Duan S, Helms CA, Liu Y, Chen Y, McBride AA, Hwu WL, Wu JY, Chen YT, Menter A, Goldbach-Mansky R, Lowes MA, Bowcock AM: PSORS2 is due to mutations in CARD14. Am J Hum Genet 2012, 90:784-795

34. Kim HJ, Barajas B, Chan RC, Nel AE: Glutathione depletion inhibits dendritic cell maturation and delayed-type hypersensitivity: implications for systemic disease and immunosenescence. J Allergy Clin Immunol 2007, 119:1225-1233

35. Ghoreschi K, Bruck J, Kellerer C, Deng C, Peng H, Rothfuss O, Hussain RZ, Gocke AR, Respa A, Glocova I, Valtcheva N, Alexander E, Feil S, Feil R, Schulze-Osthoff K, Rupec RA, LovettRacke AE, Dringen R, Racke MK, Rocken M: Fumarates improve psoriasis and multiple sclerosis by inducing type II dendritic cells. J Exp Med 2011, 208:2291-2303

36. Peng $\mathrm{H}$, Guerau-de-Arellano $\mathrm{M}$, Mehta VB, Yang Y, Huss DJ, Papenfuss TL, Lovett-Racke AE, Racke MK: Dimethyl fumarate inhibits dendritic cell maturation via nuclear factor kappaB (NFkappaB) and extracellular signal-regulated kinase 1 and 2 (ERK1/2) and mitogen stress-activated kinase 1 (MSK1) signaling. J Biol Chem 2012, 287:28017-28026

37. Aw Yeang HX, Hamdam JM, Al-Huseini LM, Sethu S, Djouhri L, Walsh J, Kitteringham N, Park BK, Goldring CE, Sathish JG: Loss of transcription factor nuclear factor-erythroid 2 (NF-E2) p45-related factor-2 (Nrf2) leads to dysregulation of immune functions, redox homeostasis, and intracellular signaling in dendritic cells. J Biol Chem 2012, 287:10556-10564

38. van Oosten BW, Killestein J, Barkhof F, Polman CH, Wattjes MP: PML in a patient treated with dimethyl fumarate from a compounding pharmacy. N Engl J Med 2013, 368:1658-1659 